\title{
Application of the Nonlinear Oscillations Theory to the Study of Non-equilibrium Financial Market
}

\author{
Nikolay Markov, Viktor Dmitriev, Svetlana Maltseva, Andrey Dmitriev
}

\author{
National Research University Higher School of Economics \\ Faculty of Business and Management, School of Business Informatics \\ 33 Kirpichnaya Str., 105187 Moscow, Russia \\ E-mail: a.dmitriev@hse.ru
}

\begin{abstract}
The research deals with the construction, implementation and analysis of the model of the non-equilibrium financial market using econophysical approach and the theory of nonlinear oscillations. We used the scaled variation of supply and demand prices and elasticity of these two variables as dynamic variables in the simulation of the non-equilibrium financial market. View of the dynamic variables data was determined based on the strength of econophysical prerequisites using the model of hydrodynamic type. As a result, we found that the non-equilibrium market can be described with a good degree of accuracy with oscillator models with nonlinear rigidity and a self-oscillating system with inertial self-excitation. The most important states of model of oscillation non-equilibrium model of the market were found, including the appearance of chaos and its mechanisms. We have made the calculations of the correlation dimension for the financial time series. The results show that all observed time series have a clearly defined chaotic dynamic nature.
\end{abstract}

Keywords: ask price, bid price, correlation dimension, financial market, financial time series, Lorenz system, low-dimensional chaos, non-equilibrium system, nonlinear oscillations

JEL codes: C610, C620, C690, G100

\section{Introduction}

Since the second half of the XX century, the general trend of science development has been the penetration of the ideas and methods of physics in natural and traditional humanities. Methods of physical modeling are often used in sciences such as demography, sociology, linguistics and economics.

In the mid-1990s there was an interdisciplinary research field, known as Econophysics, applying theories and methods originally developed by physicists in order to solve problems in economics, usually those including uncertainty or stochastic processes and nonlinear dynamics. The term "econophysics" was coined by $\mathrm{H}$. Eugene Stanley to describe the large number of papers written by physicists on the issues of (stock and other) markets (for econophysics reviews see Chakraborti et al., 2011; Richmond et al., 2013; Savoiu, 2013).

In 1988 Robert Savit presented the first studies of chaos in the financial markets (Savit, 1988). "Deterministic chaos" (or low-dimensional chaos) is a term used to denote the irregular behavior of dynamic systems arising from a strictly deterministic 
time evolution without any source of noise or external stochasticity (Korsch and Jodl, 1999). Most surprisingly, it turned out that such chaotic behavior can already be found for systems with a very low degree of freedom and it is, moreover, typical of most systems.

Since the end of the XX century there have been two fields of research devoted to the low-dimensional chaos in the financial markets. Some researchers have focused on the detection of the chaos in the financial time series using fractal dimensions (for examples, box counting dimension, information dimension and correlation dimension) as various measures of chaos (Lai and Ye, 2003). So, Frank and Stengos examined gold and silver returns and found that the correlation dimension is between 6 and 7 while the Kolmogorov entropy is about 0.2 for both assets (Murray and Stengos, 1989). In 1991 Steven Blank analyzed the futures prices for the S\&P500 index and soybeans (Blank, 1991). All of their results were consistent with those of markets with underlying generating systems characterized by deterministic chaos (they give necessary, but not sufficient, conditions to prove the existence of deterministic chaos). Gregory Decoster, Walter Labys and Douglas Mitchell searched for evidence of chaos in commodity futures (silver, copper, sugar and coffee) prices and found evidence of nonlinear structure (Decoster et al., 1992). Scott Mayfield and Bruce Mizrach estimated the dimension of the S\&P500 (sampled at approximately 20second intervals) and concluded that the data are either of low dimension with high entropy or nonlinear but of high dimension (Mayfield and Mizrach, 1992). Yang and Brorsen found evidence of nonlinearity in several futures markets, which was consistent with deterministic chaos in about half of the cases (Yang and Brorsen, 1993). Abhyankar, Copeland and Wong (1995) tested for the presence of chaos in the FTSE 100 Index using a six-month sample of about 60,000 minute-by-minute returns and found little to support the view that the process is chaotic at any frequency. Andreou, Pavlides and Karytinos (2000) examined four major currencies against GRD and found evidence of chaos in two out of four. Panas and Ninni (2000) found strong evidence of chaos in daily oil products for the Rotterdam and Mediterranean petroleum markets. Antoniou and Vorlow (2005) investigated the "compass rose" patterns revealed in phase portraits (delay plots) of FTSE 100 stock returns and found a strong nonlinear and possibly deterministic signature in the data generating processes. Other relevant works in this area are Hafner and Reznikova, (2012); Urrutia et al., (2002).

In our opinion, the most comprehensive review of mathematical models of financial markets is presented in the book by R. J. Elliott and P. E. Kopp (Elliot and Kopp, 2005). Despite the many pithy models presented in the book and various articles (Cai and Huang, 2007; Chen, 2008; Holyst et al., 2001), we could not find a mathematical model of the financial market whose solutions are changes in ask and bid prices. The importance of building such a model has various reasons, especially - the possibility of obtaining of financial time series and their further analysis and prediction.

We have built a model of the "big" market that is such a market which operates many economic agents pursuing their own respective interests and goals. We believe that the market is a system that generates aggregated factors from the combined action of individual interests and needs. In this case, economic dynamics are based on some 
basic dynamic structure. Aggregated factors are manifested on a macroscopic scale and act according to the laws of deterministic connections and relationships.

During the constructing of the model we proceeded from the assumption that the market in terms of generalized macroscopic flows of capital, goods and services in the phase space of a corresponding economic dynamic system is homeomorphic to the so-called dynamic systems of hydrodynamic type. If there are interaction counter flows in such systems, then they tend to be a phenomenon of generalized turbulence (Mantegna and Stanley, 1996) generating a crisis mode of the states of dynamic systems.

Modern stock markets are armed with powerful computer technology that allows for the management and documentation of real economic processes, which unfold in such markets in real time. Changing the conditions of such markets is very fast, and this fact allows you to receive time series, representing them on a small scale of time, which is a prerequisite for the detection of the deterministic component of dynamic processes.

Thus, the aim of the study is building a model of the non-equilibrium financial market that can generate the deterministic component of empirical time series using the econophysical assumptions and the nonlinear oscillation theory.

This paper is organized as follows. In section 1 we present the nonlinear dynamic model of the non-equilibrium financial market model, including the definition of the dynamic variables. In section 2 we present the nonlinear dynamic model of the nonequilibrium financial market model as a nonlinear oscillator. In section 3 we present the results and discussions, including the definition of the regular and the chaotic states of the financial market. In section 4 we give the numerical results of correlation dimension from the financial time series. In section 5 we conclude this paper.

\section{Construction of the Non-equilibrium Financial Market Model}

\subsection{Dynamic Variables}

We suppose that the financial market can be considered as a non-equilibrium open system (or dissipative system). In physics, a dissipative system is an open system which is operating out of, and often far from, equilibrium in an environment with which it exchanges energy and matter (Chen, 2015). Open systems have input and output flows, representing exchanges of matter, energy or information with its surroundings. The input and output flows on the financial market are the information flows and money flows. We suppose also that the financial market can be in equilibrium and non-equilibrium states. If the intensity of the external information flow is small, then the financial market is in equilibrium. If the intensity of the external information flow is large, then the financial market is in non-equilibrium.

A dynamic system can be described simply as a system of $N$ first-order differential equations 


$$
\dot{x}_{i} \equiv \frac{d x_{i}}{d t}=f_{i}\left(x_{1}, \ldots, x_{N}\right), i=1, \ldots, N
$$

where the independent variable $t$ can be read as time and the $x_{i}(t)$ are dynamic variables whose time dependence is generated by the equations, starting from specified initial conditions $x_{i}(0), i=1, \ldots, N$. It should be noted that the dynamic system is autonomous because it is not explicitly $t$-dependent (Ruelle, 1989).

Let's define the dynamic variables of the mathematical model:

- $Y_{1}(t)$ states the localized variable of the demand function,

- $Y_{2}(t)$ states the localized variable of the supply function,

- $X_{1}(t)$ states locally varying demand price,

- $X_{2}(t)$ states locally varying supply price,

- $Y_{1}^{(0)}=Y_{2}^{(0)}=Q_{0}$ states the equilibrium values of the functions of supply and demand in a market equilibrium condition,

- $X_{1}^{(0)}=X_{2}^{(0)}=P_{0}$ states the equilibrium values of the price of supply and demand in the equilibrium state of the market $R=\left(P_{0}, Q_{0}\right)$,

- $Y_{i}(t)-Q_{0} \equiv \delta Y_{i}(t) \equiv y_{i},(i=1,2)$ states variations of the volume of demand and supply near $R=\left(P_{0}, Q_{0}\right)$,

- $\quad X_{j}(t)-P_{0} \equiv \delta X_{j}(t) \equiv x_{j},(j=1,2)$ states variations of prices of supply and demand near $R=\left(P_{0}, Q_{0}\right)$,

- $F_{1}\left(x_{1}, x_{2}\right)$ states function of aggregated demand in cumulative product of the market as a function of various kinds of price,

- $F_{2}\left(x_{1}, x_{2}\right)$ states function of aggregated supply in cumulative product of the market as a function of various kinds of price.

Let's consider that $F_{1}\left(x_{1}, x_{2}\right)$ and $F_{2}\left(x_{1}, x_{2}\right)$ are continuous and differentiable in the variables $x_{1}$ and $x_{2}$ in the vicinity of the equilibrium point $R=\left(P_{0}, Q_{0}\right)$.

Let's construct a matrix of first partial derivatives of functions $F_{1}\left(x_{1}, x_{2}\right)$ and $F_{2}\left(x_{1}, x_{2}\right)$ near the point $R=\left(P_{0}, Q_{0}\right)$ :

$$
L=\left(\begin{array}{ll}
\frac{\partial F_{1}}{\partial x_{1}} & \frac{\partial F_{1}}{\partial x_{2}} \\
\frac{\partial F_{2}}{\partial x_{1}} & \frac{\partial F_{2}}{\partial x_{2}}
\end{array}\right)
$$


Given that the price variations $x_{1}$ and $x_{2}$ are small, use the $L$. Onsager's relations (Onsager, 1931) of reciprocity in matrix form:

$$
\left(\begin{array}{l}
y_{1} \\
y_{2}
\end{array}\right)=\left(\begin{array}{ll}
L_{11} & L_{12} \\
L_{21} & L_{22}
\end{array}\right)\left(\begin{array}{l}
x_{1} \\
x_{2}
\end{array}\right)
$$

where $\left\{L_{i j}\right\}$ states phenomenological matrix coefficients (1). The matrix equation (2) connects the extensive $\left(y_{1}, y_{2}\right)$ and intensive $\left(x_{1}, x_{2}\right)$ variables of the model; in terms of the thermodynamics of irreversible processes - flows and driving forces associated with data flows.

Economic dynamics of non-equilibrium market due to deviations of demand $Y_{1}(t)$ and supply $Y_{2}(t)$ from their equilibrium state $Y_{1}^{(0)}=Y_{2}^{(0)}=Q_{0}$ should induce a temporary change in the variation of prices $\left(x_{1}, x_{2}\right)$. In the first approximation, these dynamics can be represented as a system of ordinary differential equations:

$$
\left(\begin{array}{l}
\dot{x}_{1} \\
\dot{x}_{2}
\end{array}\right)=\left(\begin{array}{ll}
K_{11} & K_{12} \\
K_{21} & K_{22}
\end{array}\right)\left(\begin{array}{l}
y_{1} \\
y_{2}
\end{array}\right)
$$

where $\dot{x}_{i}$ states first order derivative with respect to time, $\left\{K_{i j}\right\}$ states the entries of dynamic market conditions.

The joint solving of equations (2) and (3) gives the equation describing the dynamics of economic and non-equilibrium dynamic system:

$$
\left(\begin{array}{l}
\dot{x}_{1} \\
\dot{x}_{2}
\end{array}\right)=\left(\begin{array}{ll}
A_{11} & A_{12} \\
A_{21} & A_{22}
\end{array}\right)\left(\begin{array}{l}
x_{1} \\
x_{2}
\end{array}\right)
$$

where $\left(\begin{array}{ll}A_{11} & A_{12} \\ A_{21} & A_{22}\end{array}\right)=\left(\begin{array}{ll}K_{11} & K_{12} \\ K_{21} & K_{22}\end{array}\right)\left(\begin{array}{ll}L_{11} & L_{12} \\ L_{21} & L_{22}\end{array}\right)$ states matrix, which determines the dynamics of such a non-equilibrium system.

\subsection{Bid Price Dynamics}

Let's proceed to the nonlinear dynamic analysis of economic system (4). If $x_{1}=0$, then $\dot{x}_{2}=A_{22} x_{2}$. Locally varying supply price $X_{2}(t)$ comes near $P_{0}$, therefore $A_{22}<0$. In this case time of relaxation $X_{2} \rightarrow P_{0}$ is $\tau_{2}=1 /\left|A_{22}\right|$. Also there should appear states $\dot{x}_{2}=0$ around the state of dynamic equilibrium $\left|x_{1}\right|=\left|x_{2}\right|$. This is possible if $\left|A_{22}\right|=A_{21}=1 / \tau_{2}$. Consequently, up to the first order values $\left(x_{1}\right.$ and $\left.x_{2}\right)$ the second equation of the system (4) takes the following form: 


$$
\dot{x}_{2}=-\frac{1}{\tau_{2}}\left(x_{2}-x_{1}\right)
$$

Similar arguments about the coefficients of the first equation of (4) lead to the fact that $A_{11}=1 / \tau_{1}$, where $\tau_{1}$ states characteristic time of relaxation $X_{1} \rightarrow P_{0}$. If $\left|x_{1}\right|$ and $\left|x_{2}\right|$ are small enough, then $A_{12}$ is constant. In this case, the solution of (3) is a noise relaxation oscillations near the equilibrium state $R=\left(P_{0}, Q_{0}\right)$. Relaxation means the return of a perturbed system into equilibrium.

According to the Le Chatelier-Braun principle (Atkins, 1993), if a system deviates from the state of the stable equilibrium, then the forces arise and try to return the system back to the equilibrium state. If $\left|X_{1}(t)-P_{0}\right| \square P_{0}$, then as a first approximation it can be considered that these forces are proportionate to deviation.

With the growth of the amplitude values of deviations $X_{1}(t)$ and $X_{2}(t)$ from $R=\left(P_{0}, Q_{0}\right)$ variations in prices of supply and demand start to "cling" to each other.There is a simple nonlinear interaction between supply and demand in the system (4). Consequently, there is a relation between variables $x_{1}$ and $x_{2}$, describing the elasticity of $x_{1}$ due to $x_{2}$. In this case, elasticity is the measurement of how responsive the ask price is to a change in the bid price.

Consider,

$$
A_{12}(t)=c E_{12}(t)
$$

where $E_{12}(t)$ - the elasticity of $x_{1}$ due to $x_{2}, c$ - parameter, used for dimensions matching. With help of choosing of measure units we can prove that $|c|=1$.

Introduce the dimensionless time $T=t / \tau_{1}$. Multiplying the equation (5) to $\tau_{1}$, we get the following equation:

$$
\frac{d x_{2}}{d T}=-\sigma\left(x_{2}-x_{1}\right)
$$

where $\sigma=\frac{\tau_{1}}{\tau_{2}}=\left|\frac{A_{22}}{A_{11}}\right|$. Factor $\sigma$ shows the relation between the relaxation speeds $X_{1} \rightarrow P_{0}$ and $X_{2} \rightarrow P_{0}$, i.e. it represents the relative sensitivity to changes in market prices of supply and demand. If $\tau_{2}<\tau_{1}$, then we can observe an effect of delay of the reaction of demand price to rapid change in the supply price, which leads to certain dynamic effects. 


\subsection{Ask Price Dynamics}

In the view of (6) and (7), the first equation of system (4) takes the following form:

$$
\frac{d x_{1}}{d T}=-x_{1}+\tau_{1} c E_{12}(t) x_{2}
$$

\subsection{Elasticity Dynamics}

The value $E_{12}(t)$ is regarded as an independent dynamic variable, provided in the following form:

$$
E_{12}(t)=E_{12}^{(0)}+\delta E_{12}(t)
$$

where $E_{12}^{(0)}$ states elasticity in the equilibrium state.

The essence of the non-equilibrium dynamics of the market leads to the conclusion that in the first nonlinear approximation, the differential equation for $E_{12}(t)$ takes the following form:

$$
\dot{E}_{12}=-e E_{12}+k x_{1} x_{2}
$$

where $e, k>0$ - constants, $e=1 / \tau_{E}, \tau_{E}$ states characteristic time of relaxation $E_{12}(t) \rightarrow E_{12}^{(0)}$

Let's introduce $C=\tau_{1} L_{21}, K=\tau_{1} k, \beta=\tau_{1} / \tau_{E}$ and multiply (10) to $\tau_{1}$. Then we get the following equation:

$$
\dot{E}_{12}=-\beta E_{12}+K x_{1} x_{2}
$$

Let's also introduce some characteristic elasticity scale $\lambda$ and new renormalized quantities:

$$
\rho=\frac{E_{12}^{(0)}}{\lambda}, z=\rho-\frac{E_{12}}{t}, x=\sqrt{\frac{K}{C}} x_{1}, y=\sqrt{\frac{K}{C}} x_{2}, \dot{x}=\frac{d x}{d T}, \dot{y}=\frac{d y}{d T}, \dot{z}=\frac{d z}{d T}
$$

Therefore the equations (7), (8) and (11) respectively take the following forms:

$$
\begin{aligned}
& \dot{x}=-\sigma(y-x) \\
& \dot{y}=\rho x-y-x z \\
& \dot{z}=-\beta z+x y
\end{aligned}
$$

The system of equations (13), (14) and (15) is well known as Lorenz system (Lorenz, 1963). 


\section{Nonlinear Oscillations}

Now let's reduce the number of equations describing the non-equilibrium market.

Introduce a new variable:

$$
u \equiv 2 \sigma z-x^{2}
$$

Taking into account (16), equation (13) takes the following form:

$$
\ddot{x}=-(\sigma+1) \dot{x}-\frac{1}{2} x^{3}+\left[\sigma(\rho-1)-\frac{1}{2} u\right] x
$$

Taking into account (16), equation (15) takes the following form:

$$
\dot{u}=-\beta u+(2 \sigma-\beta) x^{2}
$$

The system of equations (17) and (18) corresponds to the dynamic model of an oscillator with inertial nonlinear inflexibility (Hayfeh and Mook, 1995). Equation (17) represents the dynamics of inharmonic oscillations along the coordinate axis $O x$ in two-humped potential pit, damped with the member $-(\sigma+1) \dot{x}$ and described by the function

$$
V(u, x)=\frac{1}{8} x^{4}-\frac{1}{2}\left[\sigma(\rho-1)-\frac{1}{2} u\right] x^{2} \equiv \frac{1}{8} x^{4}-\frac{1}{2} \omega x^{2}
$$

The value $\omega(u)=\sigma(\rho-1)-\frac{1}{2} u$ is not a constant. Consequently, the system generates complex auto-oscillatory relations between $u(x)$ and $x(u)$, defined by equations (17) and (18).

The system of equations (17) and (18) generates a complex irregular movement in a plane of the variables $(u, x)$ that under certain relations between the parameters $\langle\sigma, \beta, \rho\rangle$ generate a phenomenon called dynamic chaos (Wiggins, 2003).

The system of equations (17) and (18) is equivalent to Lorenz system, but now it can interpreted in the following way.

First of all, let's $u=$ const. Then (17) is an equation of nonlinear oscillator $\ddot{x}+\gamma \dot{x}=-\frac{\partial V}{\partial x}$, where $\gamma=\sigma+1$ - dissipation parameter and potential function depends on $\rho, \sigma, u$ parameters and has a view (19).

In the field of parameters, corresponding with complicated chaotic dynamics, $\sigma(\rho-1)>0$, thus for small $u$ the coefficient for quadratic term is negative and potentially has two symmetrically posed minimums. For big $u$ the coefficient for quadratic term becomes positive and at least one (Fig. 1). However $u$ in the system under consideration is not a parameter, but a dynamic variable as a subject of (18). 
It controls the change of $x^{2}$ value in time, not instantly, but with inertness and smoothing that are determined by $\beta$ parameter.

Figure 1 Potential function $V(x)$

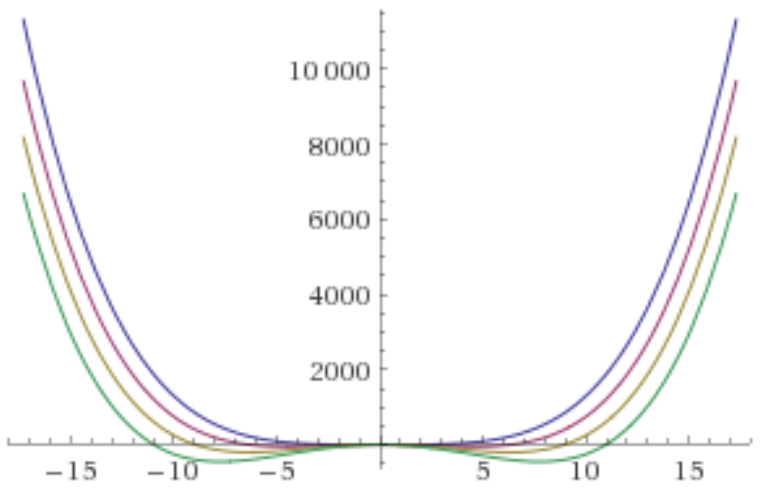

Source: WolframAlpha ${ }^{\circledR}$

System dynamics can be presented as fluctuation inside one of potential pits with occasional transfer to another one caused by $u$ parameter change and the corresponding potential form change.

\section{Results and Discussion}

\subsection{Asymptotically Stable Equilibrium Price}

Let's proceed to a qualitative analysis of the systems of differential equations (Wiggins, 2003). Let's find the stationary points and determine their stability.

The system of differential equations (17) and (18) has three stationary points:

$$
O(0,0), E_{1,2}( \pm \sqrt{\beta(\rho-1)},(2 \sigma-\beta)(\rho-1))
$$

If $\rho<1$, then the zero point is asymptotically stable. At the same time there are no other real stationary points $E_{1,2}$. Point $\rho=1$ is a point of supercritical pitchfork bifurcation. If $\rho>1$, then the zero stationary point is unstable.

Real stationary points $E_{1,2}$ appear if $\rho>1$. At the same time, if $1<\rho<\rho_{c}$, then the points are stable; if $\rho>\rho_{c}$, then $E_{1,2}$ - unstable. Here $\rho_{c}=\sigma \frac{\sigma+\beta+3}{\sigma-\beta-1}$, where $\sigma>\beta-1$. In these cases, $\beta(\rho-1) \neq 0$ and stable equilibrium market price does not exist.

The condition for the existence of equilibrium (not necessarily stable) market price is the equality of supply and demand prices $X_{1}(t)=X_{2}(t)=P_{0}$ or achieving a state of 
equilibrium system $R=\left(P_{0}, Q_{0}\right)$. In the notation of the Lorenz system for variations in the price of supply and demand, this condition is equivalent to $x=y=0$.

Consequently, if and only if $\frac{E_{12}^{(0)}}{\lambda}<1$, the market may contain asymptotically stable equilibrium price (fig. 2). It is the equilibrium state of the financial market. The real financial market is not in an equilibrium state.

Figure 2 Asymptotically stable solution $x(t)$ at $\rho=0.7$

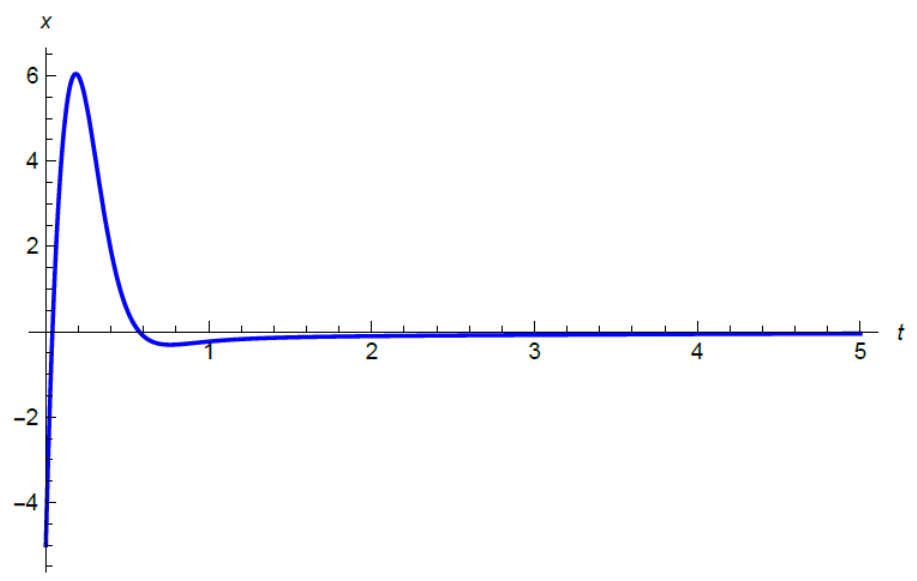

Source: Wolfram Mathematica ${ }^{\circledR} 10.2$

\subsection{Dynamic Chaos and Financial Time Series}

Let's fix $\sigma$ and $\beta$, i.e. ratio of times of relaxation $\frac{\tau_{1}}{\tau_{2}}$ and $\frac{\tau_{1}}{\tau_{E}}$. Consider $\frac{\tau_{1}}{\tau_{2}}=10$ and $\frac{\tau_{1}}{\tau_{E}}=\frac{8}{3}$. At the same time we vary the parameter $\rho=\frac{E_{12}^{(0)}}{\lambda}$.

For sufficiently large values $\rho(\rho \geq 28)$, i.e. for big elasticity $\frac{E_{12}^{(0)}}{\lambda}$, random (irregular) paths $x(t)$ and $u(t)$ appear in the system of equations (17) and (18) (fig. 3). For Lorenz system, when $T \rightarrow \infty$ phase path fills an attractive special area near points $E_{1}$ and $E_{2}$, which is called a strange attractor, in this case - the Lorenz attractor (Lorenz, 1963). Lorenz attractor is stable, i.e. it is preserved under small changes in the parameters. An attractor is a set of states (points in the phase space), invariant under the dynamics, towards which neighboring states in a given basin of attraction asymptotically approach in the course of dynamic evolution (Baker and Gollub, 1996). The Lorenz attractor has a correlation dimension of $2.05 \pm 0.01$ (Grassberger and Procaccia, 1983). For more details, see Lichtenberg and Lieberman (1983) and Tabor (1989). 
Figure 3 Chaotic solution $x(t)$ at $\rho=28$

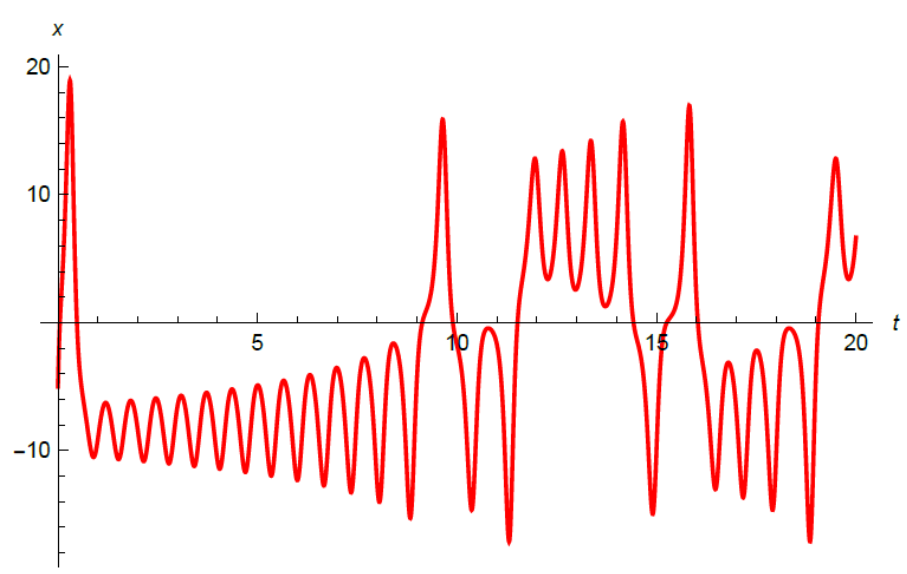

Source: Wolfram Mathematica ${ }^{\circledR} 10.2$

Strange attractors are one example of the appearance and manifestation of the evolutionary phenomenon that is called a catastrophe (Arnold, 1992). Accidents in dynamic systems give rise to such a phenomenon as dynamic chaos. One of the most difficult and important tasks - to learn how to predict occurrence of the phenomenon of dynamic chaos and precede the appearance of this kind of phenomena.

Thus, in complex dynamic systems such as financial markets, there are basic nonlinear dynamics, which generate the observed sequence of their states in its various modes of display, which are called time series. Time series that are obtained from empirical observations and that are "well" organized carry meaningful information about the deterministic component of the dynamics that form these series.

\section{Correlation Dimension of Financial Time Series}

The determination of the correlation dimension (Grassberger and Procaccia, 1983) for a supposed chaotic process directly from experimental time series is an often used means of gaining information about the nature of the underlying dynamics (see, for example, contributions in Ding et al., 1993; for reviews on dimension measurements see Grassberger et al., 1991). In particular, such analyses have been used to support the hypothesis that the time series results from an inherently low-dimensional chaotic process (Ding et al., 1993).

The geometry of chaotic attractors can be complex and difficult to describe. It is therefore useful to have quantitative characterizations of such geometrical objects. One of these characterizations is the correlation dimension $D_{2}$. The correlation dimension has several advantages comparing to other dimensional measures:

- $D_{2}$ is easy to compute from financial experimental data. 
- Using $D_{2}$ one can distinguish a chaotic dynamic system ( $D_{2}$ is finite) from a stochastic system ( $D_{2} \rightarrow \infty$ ).

- $D_{2}$ is the lower bound estimate of attractor's dimension $d\left(d \geq D_{2}\right)$.

The correlation dimension of the attractor of dynamic system can be estimated using the Grassberger-Procaccia algorithm (GP algorithm) (Grassberger and Procaccia, 1983). The implementation of GP Algorithm in this work is written in Mathematica Language for Wolfram Mathematica ${ }^{\circledR} 10.2$.

Table 1 provides summary of calculated correlation dimension for financial time series. These time series include the following:

- EUR/USD exchange rates (daily, from 04.01.1999 to 06.05.2016),

- GBP/USD exchange rates (daily, from 04.01.1971 to 06.05.2016),

- (HPQ) HP Inc. stock prices (daily, from 01.01.1995 to 06.05.2016).

Table 1 Calculated correlation dimension for financial time series

\begin{tabular}{lcc}
\hline Signal & $\begin{array}{c}\text { Data } \\
\text { Points }\end{array}$ & $\begin{array}{c}\text { Correlation } \\
\text { Dimension }\end{array}$ \\
\hline \hline EUR/USD & 4361 & 4.56 \\
\hline GBP/USD & 11372 & 4.36 \\
\hline HPQ & 5285 & 4.88 \\
\hline
\end{tabular}

Source: Authors' calculations

Based on the results, it is possible to state that currency exchange rates have chaotic dynamic nature, rather than "pure" randomness. The results show that all the observed time series have a clearly defined chaotic dynamic nature. It is also noticeable that the financial time series have the correlation dimension close to each other and equal to 4.5.

It is not strictly proven that any of the financial time series has a chaotic dynamic nature. However, we can consider the dynamic system (17) and (18) as an approximate model of the financial market. So, we also suggest that the estimation of the correlation dimension shall be a preliminary step of financial time series analysis. Moreover, the implementation of GP algorithm, including all optimizations, is computationally effective.

\section{Conclusions}

The main contributions of this paper are as follows.

- We present the nonlinear dynamic model of the financial market as a nonequilibrium system, including the definition of regular and chaotic state of the market. It is pointed out that the system is in a condition of an asymptotically stable equilibrium when the scaled elasticity is small (the ask price and bid price eventually tend to their equilibrium values).

- We report new numerical results of the correlation dimension from financial time series. The results show that all the observed time series have a clearly 
defined chaotic dynamic nature. It is also noticeable that the time series have the correlation dimension close to each other and equal to 4.5.

We found that the model of a nonlinear oscillator and the Lorenz model describe a non-equilibrium financial market. A model in the form of equations (17) and (18) gives the relationship between the demand price time, i.e. the dynamic component of financial time series. The analysis of the model suggests that market has an asymptotically stable equilibrium price at small scaled elasticity $\left(\frac{E_{12}^{(0)}}{\lambda}<1\right)$. If the scaled elasticity $\frac{E_{12}^{(0)}}{\lambda} \geq 28$, one of the crisis mode arises in the system - dynamic chaos. Given the dependence (16), these phenomena are characteristic for elasticity $E_{12}(t)$. In addition, following from the properties of the Lorenz system that models financial markets, there is asymptotically stable and chaotic dependence of the supply price from time to time.

The practical significance of the model of the financial market is associated with the solution of the problem of reconstruction of a dynamic system (Sugihara and May, 1990). In some sense, this is the inverse problem - recovering of dynamic system, using empirically observed values of financial time series, generating these financial time series. Some results of the reconstruction of a dynamic system are presented in our papers (Dmitriev et al., 2014; Maltseva et al., 2014).

\section{References}

Abhyankar, A., Copeland, L.S. and Wong, W. (1995). Nonlinear Dynamics in RealTime Equity Market Indices: Evidence from the United Kingdom. The Economic Journal, 105(431), pp. 864-880.

Andreou, A. S., Pavlides, G. and Karytinos, A. (2000). Nonlinear Time-Series Analysis of the Greek Exchange-Rate Market. International Journal of Bifurcation and Chaos, 10(7), pp. 1729-1758.

Antoniou, A. and Vorlow, C. E. (2005). Price Clustering and Discreteness: Is there Chaos behind the Noise? Physica A, 348, pp. 389-403.

Arnold, V. I. (1992). Catastrophe Theory. Springer Berlin Heidelberg.

Atkins, P. W. (1993). The Elements of Physical Chemistry, 3rd ed. Oxford University Press.

Baker, G. L. and Gollub, J. B. (1996). Chaotic Dynamics: An Introduction, 2nd Ed., Cambridge University Press.

Blank, S. (1991) "Chaos" in Futures markets? A Nonlinear Dynamical Analysis. The Journal of Futures Markets, 11(6), pp. 711-728.

Cai, G. and Huang, J. (2007). A New Finance Chaotic Attractor. International Journal of Nonlinear Science, 3(3), pp. 213-220.

Chakraborti, A., Toke, I., Patriarca, V. and Abergel, F. (2011). Econophysics review: I. Empirical facts Quantitative Finance. Quantitative Finance, 11(7), pp. 991-1012.

Chakraborti, A., Toke, I., Patriarca, V. and Abergel, F. (2011). Econophysics review: II. Agent-based models. Quantitative Finance, 11(7), pp. 1013-1041. 
Chen, J. (2015). The Unity of Science and Economics: A New Foundation of Economic Theory. Springer Berlin Heidelberg.

Chen, W. C. (2008). Dynamics and Control of a Financial System with Time-delayed Feedbacks. Chaos, Solitons and Fractals, 37(4), pp. 1188-1207.

Decoster, G. P., Labys, W. C. and Mitchell, D. W. (1992) Evidence of Chaos in Commodity Futures Prices. The Journal of Futures Markets, 12(3), pp. 291-305.

Ding, M., Grebogi, C., Ott, E., Sauer, T. and Yorke, J. (1993). Estimating correlation dimension from a chaotic time series: when does plateau onset occur? Physica $D$, 69(3-4), pp. 404-424.

Dmitriev, A. V., Maltseva, S. V. and Markov, N. V. (2014). Crisis Forecasting in Ask and Bid Prices Formation Systems in Case of Precious Metals. In: Proceedings of Global Interdisciplinary Business-Economics Advances Conference. Tampa, FI-USA, pp. 11-18.

Elliott, R. J., Kopp, P. E. (2005). Mathematics of the Financial Markets. Springer Berlin Heidelberg.

Grassberger, P., Schriber, T. and Schaffrath, C. (1991). Nonlinear time sequence analysis. International Journal of Bifurcation and Chaos, vol. 1(3), pp. 521-547.

Grassberger, P. and Procaccia, I. (1983). Measuring the trangeness of strange attractors. Physica D: Nonlinear Phenomena, 9, pp. 189-208.

Hafner, C. M. and Reznikova, O. (2012). On the estimation of dynamic conditional correlation models. Computational Statistics and Data Analysis, 56(11), pp. 35333545.

Hayfeh, A. H. and Mook, D. T. (1995).Nonlinear Oscillations. John Willey \& Sons.

Holyst, J. A., Zebrowska, M. and Urbanowicz, K. (2001). Observations of the Deterministic Chaos in Financial Time Series by Recurrence Plots, Can One Control Chaotic Economy? The European Physical Journal B, vol. 20(4), pp. 531-535.

Korsch, H. J. and Jodl, H.-J. (1999). Nonlinear Dynamics and Deterministic Chaos. Springer Berlin Heidelberg.

Lai, Y. C. and Ye, N. (2003). Recent developments in chaotic time series analysis. International Journal of Bifurcation and Chaos, 13(6), pp. 1383-1422.

Lichtenberg, A. and Lieberman, M. (1983). Regular and Stochastic Motion. Springer Berlin Heidelberg.

Lorenz, E. N. (1963). Deterministic Nonperiodic Flow. Journal of the Atmospheric Sciences, 20(2), pp. 130-141.

Maltseva, S. V. and Dmitriev, A. V. (2014). Dynamic System Crisis's Detecting Using Big Data Flow. In: Proceedings Performance Management Conference. Aarhus, Denmark, pp. 192-201.

Mantegna, R. N. and Stanley, H. E. (1996). Turbulence and Financial Markets. Nature, 383, pp. 587-588.

Mayfield, S. E. and Mizrach, B. (1992). On Determining the Dimension of Real-Time Stock-Price Data. Journal of Business \& Economic Statistics, 10(3), pp. 367-374.

Murray, F. and Stengos, T. (1989). Measuring the Strangeness of Gold and Silver Rates of Return. The Review of Economic Studies, 56(4), pp. 553-567.

Onsager, L. (1931). Reciprocal Relations in Irreversible Processes. Physical Review Letters, vol. 37, pp. 405-426.

Panas, E. and Ninni, V. (2000). Are Oil Markets Chaotic? A Non-Linear Dynamic Analysis. Energy Economics, 22(5), pp. 549-568. 
Richmond, P., Mimkes, J. and Hutzler, S. (2013). Econophysics and Physical Economics. Oxford University Press.

Ruelle, D. (1989). Elements of Differentiable Dynamics and Bifurcation Theory. Academic Press.

Savit, R. (1988). When Random is Not Random: An Introduction to Chaos in Market Prices. Journal of Futures Markets, 8(3), pp. 271-290.

Savoiu, G. (2013). Econophysics. Background and Applications in Economics, Finance, and Sociophysics. Academic Press.

Sugihara, G. and May, R. M. (1990). Nonlinear Forecasting as a Way of Distinguishing Chaos from Measurement Error in Time Series. Nature, 344, pp. 734-741.

Tabor, M. (1989). Chaos and Integrability in Nonlinear Dynamics: An Introduction. Wiley.

Urrutia, J. L., Gronewoller, P. and Hoque, M. (2002). Nonlinearity and low deterministic chaotic behavior in insurance portfolio stock returns. Journal of Risk and Insurance, 69(4), pp. 537-554.

Wiggins, S. (2003). Introduction to Applied Nonlinear Dynamical Systems and Chaos. Springer Berlin Heidelberg.

Yang, S. R. and Brorsen, D. W. (1993). Nonlinear Dynamics of Daily Futures Prices: Conditional Heteroskedasticity or Chaos? The Journal of Futures Markets, 13(2), pp. $175-191$. 